\title{
Is there a UV/X-ray connection in IRAS 13224-3809?
}

\author{
D. J. K. Buisson, ${ }^{1 \star}$ A. M. Lohfink, ${ }^{1,2}$ W. N. Alston, ${ }^{1}$ E. M. Cackett,${ }^{3}$ C.-Y. Chiang, ${ }^{3}$ \\ T. Dauser, ${ }^{4}$ B. De Marco, ${ }^{5}$ A. C. Fabian, ${ }^{1}$ L. C. Gallo, ${ }^{6}$ J. A. García, ${ }^{4,7,8}$ J. Jiang, ${ }^{1}$ \\ E. Kara, ${ }^{9}$ M. J. Middleton, ${ }^{10}$ G. Miniutti, ${ }^{11}$ M. L. Parker, ${ }^{1}$ C. Pinto, ${ }^{1}$ P. Uttley, ${ }^{12}$ \\ D. J. Walton ${ }^{1}$ and D. R. Wilkins ${ }^{13}$ \\ ${ }^{1}$ Institute of Astronomy, University of Cambridge, Madingley Road, Cambridge CB3 OHA, UK \\ ${ }^{2}$ Department of Physics, Montana State University, Bozeman, MT 59717-3840, USA \\ ${ }^{3}$ Department of Physics \& Astronomy, Wayne State University, 666 W. Hancock St, Detroit, MI 48201, USA \\ ${ }^{4}$ Dr. Karl Remeis-Observatory and Erlangen Centre for Astroparticle Physics, Sternwartstr 7, D-96049 Bamberg, Germany \\ ${ }^{5}$ Nicolaus Copernicus Astronomical Center, Polish Academy of Sciences, Bartycka 18, PL-00-716 Warsaw, Poland \\ ${ }^{6}$ Department of Astronomy and Physics, Saint Mary's University, 923 Robie Street, Halifax, NS B3H 3C3, Canada \\ ${ }^{7}$ Cahill Center for Astronomy and Astrophysics, California Institute of Technology, Pasadena, CA 91125, USA \\ ${ }^{8}$ Harvard-Smithsonian Center for Astrophysics, 60 Garden St, Cambridge, MA 02138, USA \\ ${ }^{9}$ Department of Astronomy, University of Maryland, College Park, MD 20742-2421, USA \\ ${ }^{10}$ Department of Physics and Astronomy, University of Southampton, Highfield, Southampton SO17 1BJ, UK \\ ${ }^{11}$ Centro de Astrobiología (CSIC-INTA), Dep. de Astrofísica, ESAC, PO Box 78, Villanueva de la Cañada, E-28691 Madrid, Spain \\ ${ }^{12}$ Anton Pannekoek Institute, University of Amsterdam, Science Park 904, NL-1098 XH Amsterdam, the Netherlands \\ ${ }^{13}$ Kavli Institute for Particle Astrophysics and Cosmology, Stanford University, 452 Lomita Mall, Stanford, CA 94305, USA
}

Accepted 2017 December 22. Received 2017 December 18; in original form 2017 October 2

\begin{abstract}
We present results from the optical, ultraviolet, and X-ray monitoring of the NLS1 galaxy IRAS 13224-3809 taken with Swift and XMM-Newton during 2016. IRAS 13224-3809 is the most variable bright AGN in the X-ray sky and shows strong X-ray reflection, implying that the X-rays strongly illuminate the inner disc. Therefore, it is a good candidate to study the relationship between coronal X-ray and disc UV emission. However, we find no correlation between the X-ray and UV flux over the available $\sim 40 \mathrm{~d}$ monitoring, despite the presence of strong X-ray variability and the variable part of the UV spectrum being consistent with irradiation of a standard thin disc. This means either that the X-ray flux which irradiates the UV emitting outer disc does not correlate with the X-ray flux in our line of sight and/or that another process drives the majority of the UV variability. The former case may be due to changes in coronal geometry, absorption or scattering between the corona and the disc.
\end{abstract}

Key words: accretion, accretion discs-black hole physics-galaxies: individual: IRAS13224-3809-galaxies: Seyfert.

\section{INTRODUCTION}

AGN are the most luminous persistent point sources in the Sky in the optical to X-ray bands. They have a significant impact on galaxy evolution and are therefore of great interest for study. Since AGN are unresolved with current instruments in the X-ray band, their structure must be inferred from properties of their spectra or the variability of their emission.

A significant fraction of their bolometric luminosity is emitted in the X-ray band from a small region known as the corona (Haardt \& Maraschi 1993; Merloni \& Fabian 2003). Microlensing (Dai et al. 2010; Chartas et al. 2012) and timing (De Marco et al. 2011, 2013;

^E-mail:djkb2@cam.ac.uk
Reis \& Miller 2013; Kara et al. 2014, 2016) results show that this is often smaller than $10 r_{g}$ in size. Much of the X-ray power from the corona is directed towards the accretion disc, as seen in reflection features in the X-ray spectrum (Tanaka et al. 1995; Fabian \& Ross 2010).

Additional evidence that the X-ray emission affects the disc is that variations in X-ray and optical fluxes are often seen to be correlated (e.g. Alston, Vaughan \& Uttley 2013; Shappee et al. 2014; Edelson et al. 2015; Fausnaugh et al. 2016; Buisson et al. 2017; Gliozzi et al. 2017; Lobban et al. 2018). Where the optical emission lags the X-rays, this is often interpreted as heating of the disc by the additional X-ray flux directed towards the disc (Lightman \& White 1988). In some cases (e.g. Troyer et al. 2016; Edelson et al. 2017), the lags are longer than predicted for a standard thin disc (Shakura \& Sunyaev 1973) and the X-ray light curve does not always match 
the inferred driving light curve (Starkey et al. 2017). This may be explained by a larger disc or an additional stage of reprocessing (Gardner \& Done 2017; Edelson et al. 2017). There is also now good evidence that diffuse continuum emission from the broad line region can also contribute significantly to the lags, which needs to be accounted for (Cackett et al. 2017; McHardy et al. 2017). Sometimes, the optical emission is found to lead the X-ray emission (Arévalo et al. 2005), which is interpreted as Compton upscattering of the optical photons to X-rays (Haardt \& Maraschi 1991) or the propagation of fluctuation inwards through the disc (Lyubarskii 1997; Arévalo \& Uttley 2006). However, sometimes no correlation is found (e.g. Robertson et al. 2015). Continued study of optical to $\mathrm{X}$-ray variability in more sources has the potential to provide more information on why correlations are seen only in some sources.

The narrow line Seyfert 1 (NLS1) galaxy IRAS 13224-3809 $\left(z=0.066, M_{\mathrm{BH}}=10^{6}-10^{7} \mathrm{M}_{\odot} ;\right.$ Zhou \& Wang 2005) is the most variable AGN in X-rays, often showing changes in X-ray flux by a factor of 50 on time-scales of less than $1 \mathrm{~h}$ (Boller et al. 1997; Dewangan et al. 2002; Fabian et al. 2013). Its X-ray spectrum shows a soft continuum with strong relativistic reflection and soft excess (Ponti et al. 2010; Fabian et al. 2013; Chiang et al. 2015, Jiang et al., submitted). The soft X-ray continuum suggests that IRAS $13224-3809$ is accreting at a high Eddington fraction $(\dot{m} \simeq$ 0.7 using the relation from Shemmer et al. 2008). It shows little $\mathrm{X}$-ray obscuration, although the recent XMM-Newton observations have allowed the detection of an Ultra-Fast Outflow (UFO) which is observed only at low X-ray flux (Parker et al. 2017a,b). Previous studies show that IRAS 13224-3809 has little absorption in the UV and that the C IV emission line is asymmetric and blueshifted (Leighly \& Moore 2004; Leighly 2004), which may indicate an outflow out of the line of sight.

The strong X-ray variability and reflection suggest strong variable heating of the disc, so IRAS 13224-3809 is an ideal candidate to study UV/X-ray relations. The source is a member of the sample studied in Buisson et al. (2017) to find UV/X-ray relations. This work found a marginally significant ( $2 \sigma$ confidence) lag of $U M 2$ band $(\sim 2170 \AA$ ) behind X-ray emission, suggesting that X-ray reprocessing may occur in this source. Here, we present the results from the optical monitor (OM) of the recent $1.5 \mathrm{Ms} X M M-$ Newton observing campaign of IRAS 13224-3809, along with associated Swift monitoring (50 ks XRT exposure over the period July 7 to August 14).

The increase in data now available allows us to study more of its properties. The additional Swift monitoring allows us to measure the optical/UV variable spectrum and the extensive $X M M-N e w t o n$ coverage provides constraints on the short time-scale UV/X-ray relation.

\section{OBSERVATIONS AND DATA REDUCTION}

\subsection{XMM-Newton}

We use XMM-Newton (Jansen et al. 2001) data from the recent very large programme (P.I. Fabian) dedicated to monitoring IRAS 13224-3809, with observations from 2016 July to August. Here, we consider X-ray light curves from the EPIC-pn (Strüder et al. 2001) instrument and ultraviolet light curves from the OM (Mason et al. 2001). To provide continuous coverage, the OM observations were taken in the $W 1$ band throughout and used a typical frametime of $2700 \mathrm{~s}$.

The pn data were reduced using the standard task EPCHAIN, using a 50 arcsec circular source region and an annular background region

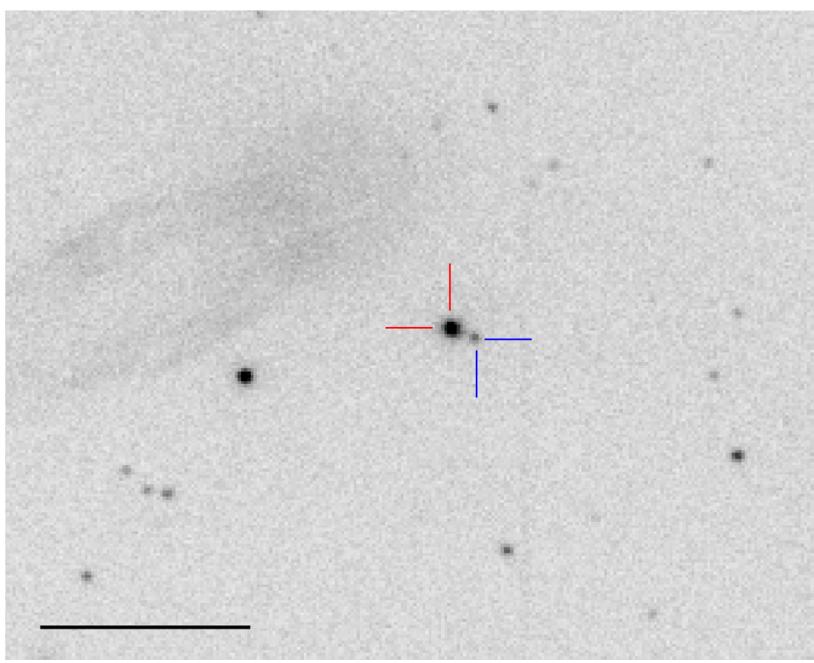

Figure 1. Image from XMM-Newton-OM showing IRAS 13224-3809 (red, left) and nearby secondary source (blue, right). The scalebar indicates 1 arcmin.

comprising radii from 60 to 90 arcsec. Data were taken in Large Window mode, leading to mild pile-up in the brightest X-ray states. While this may affect the detail of the X-ray spectra, the pile-up is too weak to have a significant impact on the work presented here ( $<15$ per cent flux loss at the light-curve peaks). Additionally, since pile-up is roughly proportional to flux, any effect on correlation measurements is minor. Light curves were produced with EVSELECT and EPICLCCORR and rebinned to match the cadence of the OM frames.

The OM photometry of IRAS $13224-3809$ is complicated by a nearby ( 7.5 arcsec separation) source (see Fig. 1) which causes the default execution of XMMEXTRACTOR to fail. We therefore take count rates directly from the images using the photometry tool IMEXAM from ZHTOOLS, extracting counts from within an aperture of radius 3 arcsec, using a nearby source-free circular region of radius 18 arcsec for background subtraction. We correct the count rates for deadtime and coincidence losses using the factors given by OMICHAIN. These corrections are between 1.043 and 1.049 for all points apart from one which is 1.029 .

In 18 exposures, a count rate less than 0 is returned, which we exclude - the sky coordinates on these images are wrong (part of OBSID 0792180501). One further point in OBSID 0792180201 is unreasonably low (about four times less than neighbouring points) so it is also excluded. This leaves 524 good OM exposures.

We also produce a light curve of the nearby source to ensure that it does not affect our results. To minimize the effect of stray light from the edges of the PSF of IRAS 13224-3809, we use a 2 arcsec radius circular aperture. This shows that the nearby source is too faint and insufficiently variable to affect the light curves of IRAS 13224-3809: its flux is 10 percent of IRAS 13224-3809 and its variability is consistent with Poisson noise.

\subsection{Swift}

Swift UVOT (Gehrels et al. 2004; Roming et al. 2005) light curves were extracted from level II image files using the tool UVOTSOURCE. We used a circular source region of 5 arcsec radius and a circular background region of 15 arcsec radius from a nearby source free area of the detector. We excluded exposures where the source region overlaps areas of the detector known to produce low count readings (Edelson et al. 2015). The good exposures are then summed across a 
whole observation. We converted count rates to fluxes using the conversion factors in Poole et al. (2008). UV fluxes were corrected for Galactic reddening using $E(B-V)=0.0601$ (Kalberla et al. 2005). We use light curves from all UVOT filters apart from the $B$ band, since the three observations available in this band are insufficient to produce reliable variability measurements.

Swift XRT (Burrows et al. 2005) light curves covering the 0.3$10 \mathrm{keV}$ energy band were produced using the online tool available on the UK Swift website ${ }^{1}$ (Evans et al. 2007, 2009). The XRT was operated in PC mode. The source region is a circle of radius 1.2 arcmin. The background region is an annulus with radii from 2.3 to 7 arcmin (with point sources removed).

The light curves from all instruments are shown in Fig. 2.

Except where noted, errors are given at the $1 \sigma$ level.

\section{RESULTS}

\subsection{Mean SED}

We show the mean SED of IRAS 13224-3809 in Fig. 3. The UV points show the mean flux across the full Swift light curve; the X-ray points show the mean spectrum from Jiang et al. (submitted). We characterize the UV spectrum with a power law of the form $f_{\lambda} \propto$ $\lambda^{\alpha}$ and exclude the $V$ band since Vanden Berk et al. (2001) show that there is a strong break in power-law index at around $5000 \AA$, blueward of the $V$ band. This gives $\alpha=-1.2 \pm 0.1$, slightly softer than the mean quasar spectrum $(\alpha=-1.56)$ found in Vanden Berk et al. (2001), suggesting that there is some contribution from the host galaxy.

From the simultaneous optical to X-ray SED, we can estimate the bolometric luminosity. We approximate the intrinsic AGN emission as a thin disc (for the UV) plus a hot blackbody (for the X-ray soft excess) and a power law (for the hard X-ray component). The principal source of uncertainty is the disc temperature, which is poorly constrained as the cut-off lies in the unobserved extreme $\mathrm{UV}$; we take the lower limit as measured from the RMS spectrum (see Section 3.2) and use the X-ray emission to provide the upper limit. This gives a bolometric luminosity range of $4 \times 10^{44}$ $1.3 \times 10^{45} \mathrm{erg} \mathrm{s}^{-1}$. For $M_{\mathrm{BH}}=10^{6}-10^{7} \mathrm{M}_{\odot}$, this implies an Eddington fraction $\dot{m}=0.3-10$. While this is not a strong constraint (due largely to the poorly determined black hole mass), a high Eddington fraction is widely regarded as typical of NLS1s and agrees well with estimates of the Eddington fraction from other methods, such as $\dot{m} \simeq 0.7$ using the $\Gamma-\dot{m}$ relation of Shemmer et al. (2008).

We also consider the relative X-ray and UV power, using the standard measure $\alpha_{\text {OX }}$ (e.g. Vagnetti et al. 2010). This gives $\alpha_{\mathrm{OX}}=-1.46$, which is compatible with (though at the X-ray weak end of) values found by various authors who have presented a $L_{\mathrm{UV}}-$ $\alpha_{\mathrm{OX}}$ relation $\left(\alpha_{\mathrm{OX}}=-1.18\right.$, Gibson, Brandt \& Schneider 2008; $\alpha_{\mathrm{OX}}=-1.31$, Grupe et al. 2010; $\alpha_{\mathrm{OX}}=-1.46$, Xu 2011).

\subsection{Swift variable UV spectrum}

To characterize the emission of the innermost regions, we study the variable part of the UV spectrum to avoid contamination by the host galaxy.

We characterize the variable part of the spectrum with the error corrected RMS flux variability, $f_{\lambda, \mathrm{Var}}=\sqrt{\sigma^{2}-\bar{\epsilon}^{2}}$ (Nandra et al. 1997; Edelson et al. 2002) as in Buisson et al. (2017), taking the

\footnotetext{
${ }^{1}$ http://www.swift.ac.uk/user_objects/
}

measured standard deviation, $\sigma$, and mean square error, $\bar{\epsilon}^{2}$, from the whole light curve. Errors on this quantity are given by $\operatorname{err}\left(f_{\lambda \text {,Var }}^{2}\right)=$ $\frac{1}{\sqrt{N}} \sqrt{\left(\sqrt{2} \bar{\epsilon}^{2}\right)^{2}+\left(2 \sqrt{\bar{\epsilon}^{2}} f_{\lambda, \text { Var }}\right)^{2} \bar{x}^{2}}$ (Vaughan et al. 2003).

The spectrum this produces (Fig. 4) is consistent $\left(\chi^{2} /\right.$ d.o.f. $\left.=2.8 / 3\right)$ with a power law, $f_{\lambda} \propto \lambda^{\alpha}$, with index $\alpha=-2.67 \pm 0.15$ (or in frequency units, $f_{v} \propto v^{\beta}$, with $\beta=0.67 \pm 0.15$ ). This is consistent at $2 \sigma$ with the expected index for the emission produced by irradiation of a thin disc $(\alpha=-2$ to $-2.33, \beta=0$ to 0.33 , Davis, Woo \& Blaes 2007) and significantly flatter than the Rayleigh-Jeans tail of a single-temperature blackbody $(\alpha=-4, \beta=2)$.

Since the variability is expected to originate in a disc spectrum, we also test a power law with an exponential cut-off representing the maximum temperature at the inner edge of the disc. This places an upper limit of $1440 \AA$ on the cut-off (at 90 per cent confidence), corresponding to a blackbody temperature of $\geq 10^{5} \mathrm{~K}$. However, such a low cut-off requires a steeper power-law index, $\alpha=-4$. This limit to the temperature is less than that predicted for a standard disc (Shakura \& Sunyaev 1973), even for conservative parameters for IRAS $13224-3809\left(M_{\mathrm{BH}}=10^{7} \mathrm{M}_{\odot}, \dot{m}=0.1\right)$, so the spectrum is consistent with the temperature of a standard disc.

\subsection{Short time-scale optical variability}

While the UVOT light curve shows that IRAS 13224-3809 varies over the course of the observing campaign, we also seek to characterize that UV variability on shorter time-scales with the $X M M$ Newton-OM data.

We calculate the average power spectral density (PSD; Vaughan et al. 2003) of the optical monitor data over the whole observation. Since calculating the PSD requires an evenly sampled time series, we split the observations where consecutive points are separated by more than 1.5 times the average. We then take sections of $120 \mathrm{ks}$ and linearly interpolate on to a regular time grid. We calculate the periodogram for each section separately and average these into frequency bins containing at least 20 points to give the PSD. This is shown in Fig. 5. The expected Poisson noise level is calculated from equation A3 in Vaughan et al. (2003) and shown as the dashed line in Fig. 5. We also fit the PSD with a sum of power-law red noise and Poisson white noise. The resulting parameters are shown in Table 1. The shape of the power spectrum is dependent on the assumptions made about Poisson noise, so we cannot simultaneously constrain the shape of the power spectrum and the level of Poisson noise, which only dominates at higher frequencies. Owing to the large uncertainties, there is insufficient statistical evidence to choose one model over another. We expect that fixing the Poisson noise to the calculated value gives the most reliable intrinsic PSD shape, $P(f) \propto f^{1.3 \pm 0.3}$. Independent of the exact model chosen, the UV PSD shows that the UV variability has the form of red noise on short time-scales.

\subsection{X-ray/UV correlation}

To study the link between the emission from the accretion disc and coronal X-ray emission, we search for correlations between UV and $\mathrm{X}$-ray flux in the XMM-Newton observations.

Initially, we produce a flux-flux plot (Fig. 6) to detect correlations between simultaneous X-ray and UV emission. This shows no strong correlation between the two bands, with Pearson coefficient $r=-0.02$ ( $r=0.025$ in the logarithmic domain) when using the full $0.3-10 \mathrm{keV}$ band. When drawing light curves from uncorrelated 

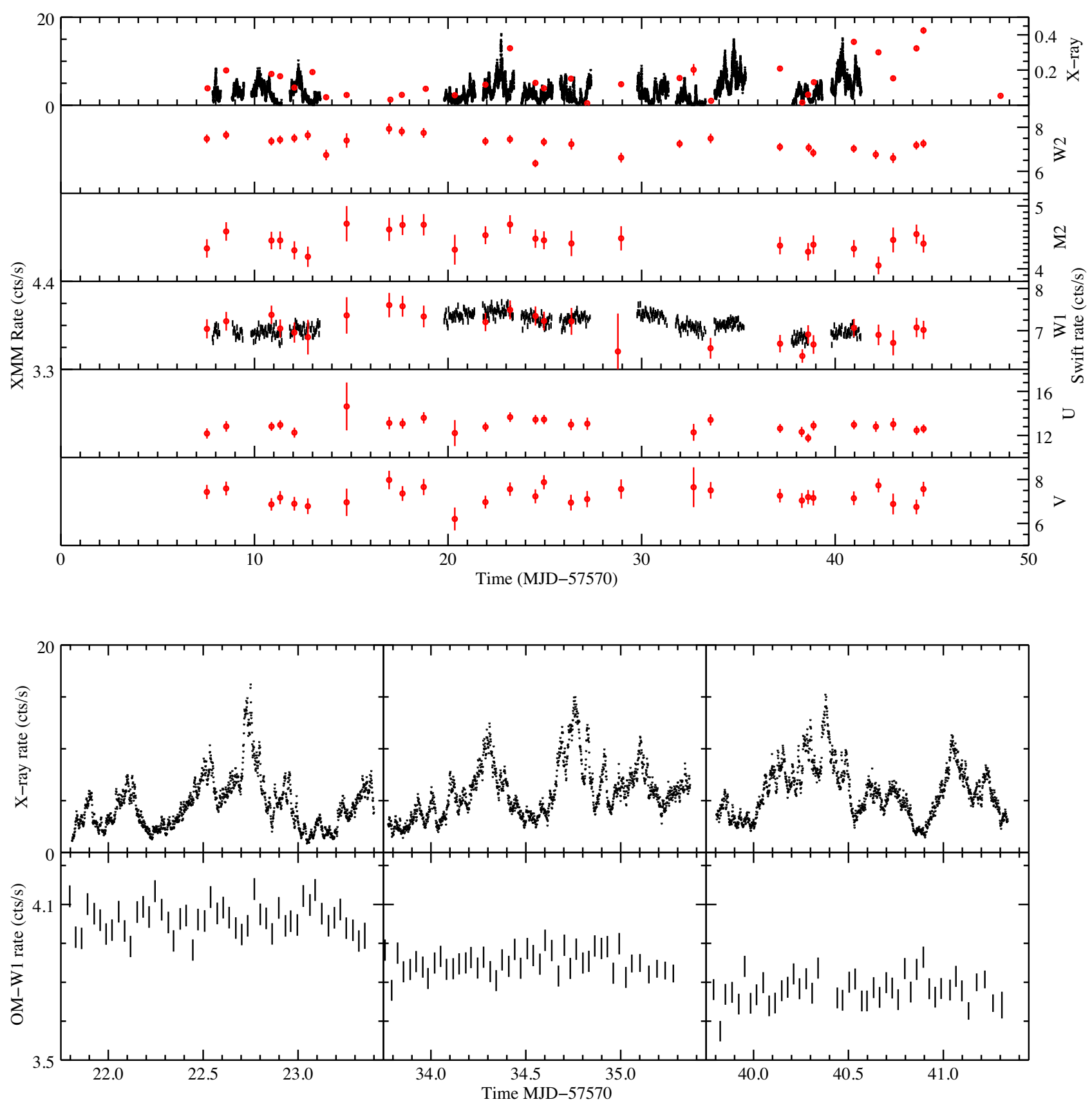

Figure 2. Light curves of IRAS 13224-3809 from XMM-Newton (black) and Swift (red). Upper panels show, from top to bottom: X-rays (0.3-10 keV), W2 band, $M 2$ band, $W 1$ band, $U$ band, $V$ band. Note that the W1 filters of Swift and XMM-Newton, although plotted in the same panel, are not identical. Lower panels show detail of the X-ray and UV light curves of the three XMM-Newton orbits with the strongest X-ray peaks. There is no apparent response of the UV emission to the $\mathrm{X}$-ray peaks.

red noise [from the same power spectra used for the discrete crosscorrelation function (DCF) simulations presented below], a stronger correlation occurs with probability $p=0.95(0.94)$. To determine whether the UV correlates with only the primary continuum (rather than the soft excess or reflected emission), we also consider the 2$4 \mathrm{keV}$ band, which is dominated by the primary emission. This also shows no correlation $(r=-0.15, p=0.61 ; r=-0.17, p=0.60$ logarithmically) with the UV.

To test whether the lack of correlation seen in the flux-flux plot is due to a lag between X-ray and UV emission, we use the DCF ( Edelson \& Krolik 1988) from a single light curve of the whole observation so that time-scales up to the full length of the observation are included.

To assess the significance of any correlations, we simulated 10000 pairs of uncorrelated light curves and estimated 95 and 99 per cent confidence intervals from the DCFs measured from these light curves. We used the method of Timmer \& Koenig (1995) to generate light curves with appropriate red-noise power spectra. Since the shape of the UV PSD is poorly constrained, we use a simple power law with $P(f) \propto f^{-\alpha}$ with $\alpha=2$ for the UV. For the $\mathrm{X}$-rays, we use a broken power law with $\alpha=1.1$ and 2.22 below and above $6 \times 10^{-5} \mathrm{~Hz}$ respectively for the X-rays (Alston et al., in 


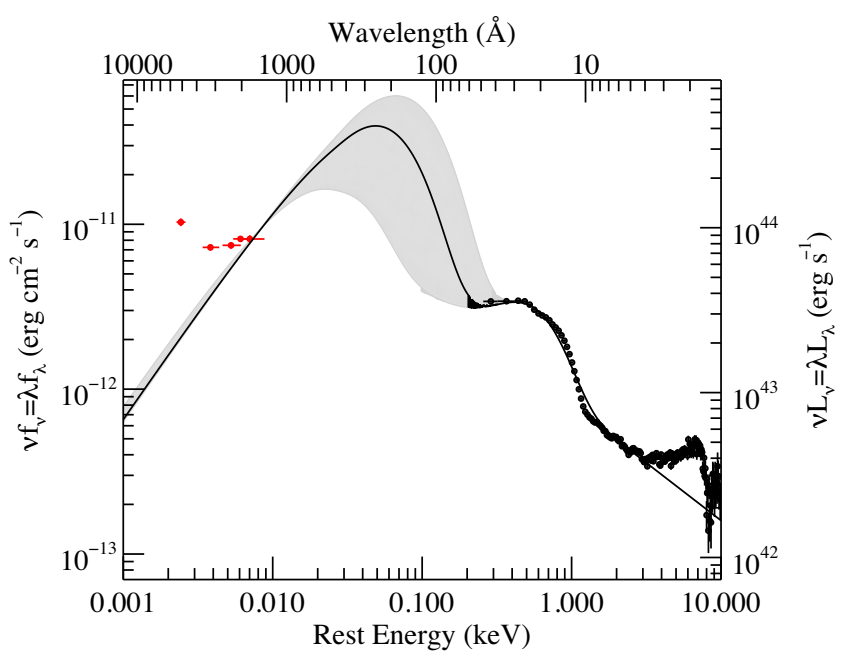

Figure 3. Mean SED of IRAS 13224-3809. Optical/UV points (red) are from Swift UVOT; X-ray points (black) are from XMM-Newton-pn (Jiang et al. submitted). The grey region indicates the range of SED models used to derive the bolometric luminosity.

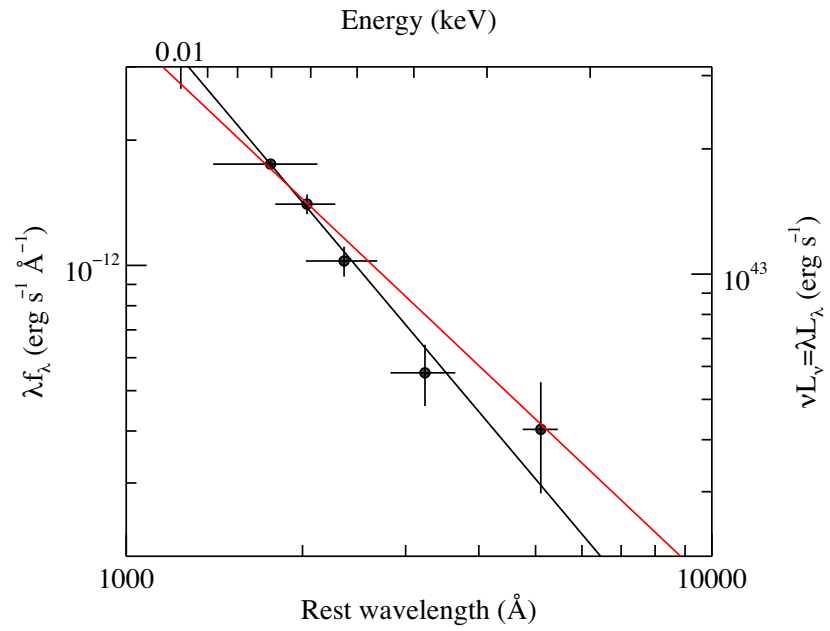

Figure 4. RMS spectrum from Swift UVOT data. The black line shows a power-law fit, with index $\alpha=-2.67 \pm 0.15$. The red line has the index expected of a thin disc, $\alpha=-2.33$. Errors in wavelength represent the half-maximum of the filters.

preparation). We extract count rates at times corresponding to the real observations and draw our final simulated data from a Poisson distribution with mean equal to the simulated rates multiplied by the frame time.

The DCF is shown in Fig 7. There are no significant correlations between the X-ray and optical monitor data. Possible anticorrelations are detected at +3.5 and $-2.5 \mathrm{~d}$, although, since there is little physical motivation for such anticorrelations, these may be sampling artefacts due to the gaps between XMM-Newton orbits. A

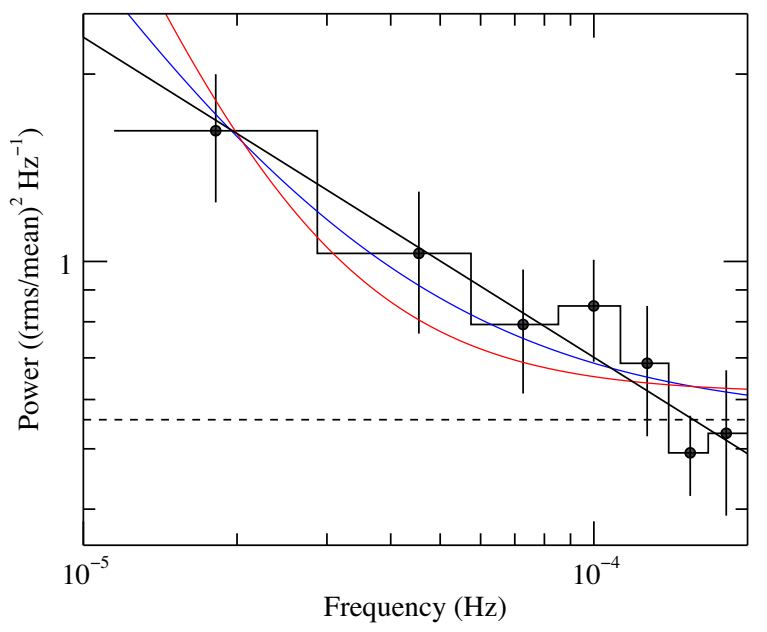

Figure 5. PSD of optical monitor data. The estimated Poisson noise level is shown by the dashed line. Solid lines show fits with a power law plus noise model, $P(f)=\alpha f^{\beta}+C$. Red: fixed index $(\beta)$, free noise $(C)$. Blue: free index, fixed noise. Black: both free. See Table 1 for full parameters.

spurious detection is not unlikely as 5 percent of points are expected to lie outside the 95 percent confidence interval. To test whether only some components of the X-ray emission are correlated with the UV, we test different X-ray bands to isolate the soft excess and power-law components; this produces similar results so we show the full band to maximize signal.

\section{DISCUSSION}

AGN almost universally show variability in their optical to X-ray spectra (e.g. Cackett, Horne \& Winkler 2007; Ponti et al. 2012). Typically, the UV and X-ray emission is seen to correlate, with the UV often lagging the X-rays, indicative of reprocessing (e.g. Edelson et al. 2015; McHardy et al. 2016; Buisson et al. 2017).

We have found that for IRAS 13224-3809, the variability in the UV emission does not clearly correlate with variability observed in X-rays. This lack of correlation is unusual but not unique: for example, 1H 0707-495, which has a similar X-ray spectrum to IRAS 13224-3809 (Fabian et al. 2009), also shows no correlation between X-ray and UV emission (Robertson et al. 2015). While these non-detections use XMM-Newton-OM monitoring covering shorter time-scales than are achievable with missions such as Swift, $\mathrm{X}$-ray reprocessing should be detectable in the XMM-Newton campaigns: there is strong X-ray variability observed on time-scales much shorter than the monitoring campaign. Indeed, UV/X-ray correlations have been detected with XMM-Newton for other sources (e.g. McHardy et al. 2016) and in shorter Swift campaigns (e.g. Edelson et al. 2017; McHardy et al. 2017; Pal \& Naik 2018). Additionally, AGN cover a wide range of black hole mass and the time-scale for variability processes scales linearly with $M_{\mathrm{BH}}$. Therefore, time-scales probed with Swift campaigns for large $M_{\mathrm{BH}}$ (e.g.

Table 1. Fits to the PSD of the XMM-Newton-OM light curve with a power law plus noise model, $P(f)=\alpha\left(f / 10^{-4} \mathrm{~Hz}\right)^{\beta}+C$.

\begin{tabular}{lcccc}
\hline Model & Norm $(\alpha)$ & Index $(\beta)$ & Noise $(C)$ & $\chi^{2} /($ d.o.f.) \\
\hline Fixed noise & $0.13 \pm 0.06$ & $-1.3 \pm 0.3$ & 0.557 & $5.68 / 5$ \\
Fixed index & $0.040 \pm 0.013$ & -2.0 & $0.61 \pm 0.05$ & $8.10 / 5$ \\
Free & $0.70 \pm 0.050$ & $-0.5 \pm 0.1$ & $<0.35$ & $2.45 / 4$ \\
\hline
\end{tabular}




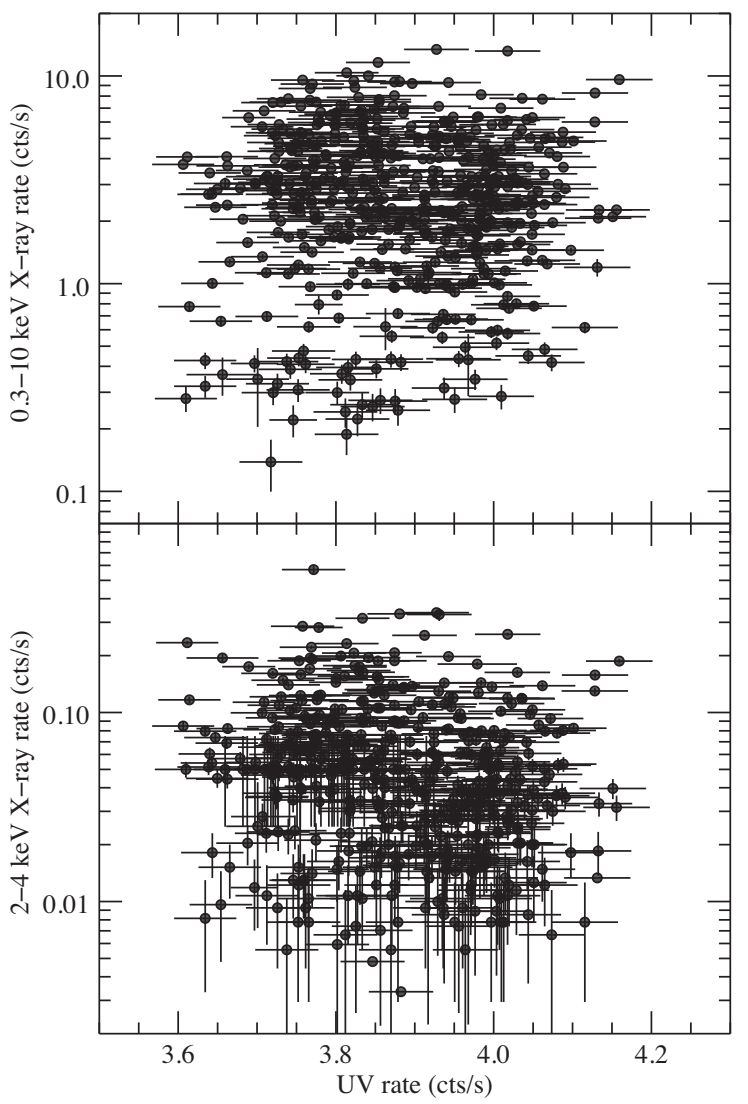

Figure 6. Flux-flux plot of X-rays (top: $0.3-10 \mathrm{keV}$, full band; bottom: 2-4 keV, power-law dominated) against UV (XMM-OM W1, $2910 \AA$ ). No correlation between the two bands is apparent.

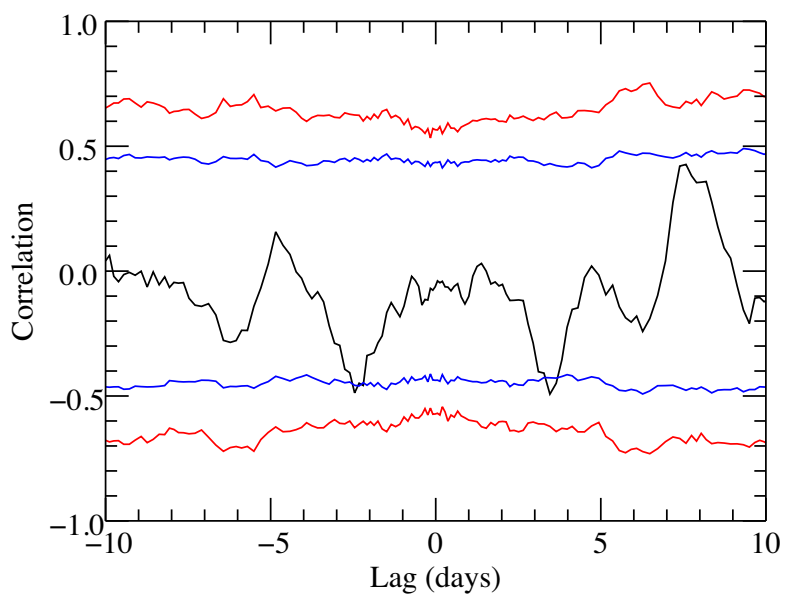

Figure 7. DCF of X-rays (0.3-10 keV) against UV (XMM-OM W1, $2910 \AA$ ). Blue and red lines indicate 95 and 99 percent confidence intervals around 0 correlation.

FAIRALL 9, where correlations are observed Lohfink et al. 2014; Buisson et al. 2017) are equivalent to the time-scales probed here. Sources that do not show UV/X-ray correlations must have different emission to typical AGN in one or both of the UV and X-ray bands.

One possibility for the lack of UV/X-ray correlation is that there are significant sources of UV variability other than X-ray irradiation. This is likely to occur in some AGN as Uttley et al. (2003) found more fractional variability in the optical than X-ray emission in
NGC 5548 (although in this case the optical and X-ray variability was correlated). One such source is the intrinsic disc fluctuations which propagate inwards to produce the $\mathrm{X}$-ray variability. However, at the radii which produce the $W 1$-band emission, the characteristic time-scale of these fluctuations is much longer than the observations analysed here.

If the lack of correlation is due to an extra source of UV variability, the UV variability would be expected to be larger than in typical AGN. On time-scales comparable to a night $\left(2 \times 10^{-5}\right.$ $2 \times 10^{-4} \mathrm{~Hz}$ ), we find variability of $0.4 \pm 0.1$ percent, consistent with Young et al. (1999), who found an upper limit on the optical variability of 1 percent within a night. We can also make a direct comparison between the fractional variability of IRAS 13224-3809 and 1H 0707-495. Robertson et al. (2015) present the fractional variability of 1H 0707-495 in two sets of four continuous orbits. To compare the same time-scales, we consider the four consecutive XMM-Newton orbits of IRAS 13224-3809 with OBSIDs 0780561501-0780561801 (other sections of consecutive orbits give similar results). This epoch has, in the $W 1$ band, $F_{\text {Var }}=1.0 \pm 0.1$ percent. These values are very similar to those of $1 \mathrm{H} 0707-495$, being between the values for the two epochs presented in Robertson et al. (2015).

Therefore, both IRAS 13224-3809 and 1H 0707-495 show only modest UV variability, close to the average of 1.2 per cent found by Smith \& Vaughan (2007) for a sample of AGN measured with the optical monitor. The similarity of the UV variability in both these sources to sample averages (e.g. Grupe et al. 2010) may suggest that it is the nature of their X-ray rather than UV variability which prevents the detection of UV/X-ray correlations.

Despite the lack of UV/X-ray correlation, the variable part of the UV spectrum has the shape expected of an irradiated disc, as found for a number of other AGN in Buisson et al. (2017) (note that while IRAS 13224-3809 was included in this paper, the Swift data at the time of writing were insufficient to produce a RMS spectrum). This also suggests that the lack of UV/X-ray correlation may be due to unusual X-ray rather than UV emission. To further constrain the nature of the UV/optical emitting region, it would be desirable to study interband UV/optical lags, which are sometimes seen to match thin disc expectations even when X-ray lags do not (e.g. Edelson et al. 2017). However, the available Swift data are insufficient to constrain these lags.

For X-ray heating to be a plausible mechanism to drive the UV variability, there must be sufficient X-ray power to cause the observed changes in UV flux. To determine the regions responsible for W1-band emission in IRAS 13224-3809, we consider a thin disc (Shakura \& Sunyaev 1973) illuminated by a central X-ray source (Cackett et al. 2007). With sensible parameters for the mass $\left(M_{\mathrm{BH}}=10^{7} \mathrm{M}_{\odot} ;\right.$ Zhou \& Wang 2005) and accretion rate $(\dot{m}=0.7$; Buisson et al. 2017; Jiang et al. submitted), we show the radii responsible for the W1-band emission in Fig. 8. To demonstrate the potential effect of X-ray irradiation, we also test the same model illuminated by an isotropic point source (Cackett et al. 2007) at $10 r_{\mathrm{G}}$ above the disc, with power $10^{44} \mathrm{erg} \mathrm{s}^{-1}$ (based on the continuum model in Jiang et al., submitted). This shows that the majority of the flux in the $W l$ band is produced on scales of a few hundred $r_{\mathrm{g}}$. The change in flux due to heating occurs slightly further out, as more significant flux changes occur when disc material is heated to temperatures at which the material starts to emit in the $W 1$ band. Integrating the flux density across the disc shows that the X-ray illumination changes the $W 1$-band flux by $v F_{v}(W 1)=2.5 \times 10^{-13} \mathrm{erg} \mathrm{s}^{-1}$. While there is significant uncertainty in some of these parameters, this shows that the effect of X-ray heating can be 


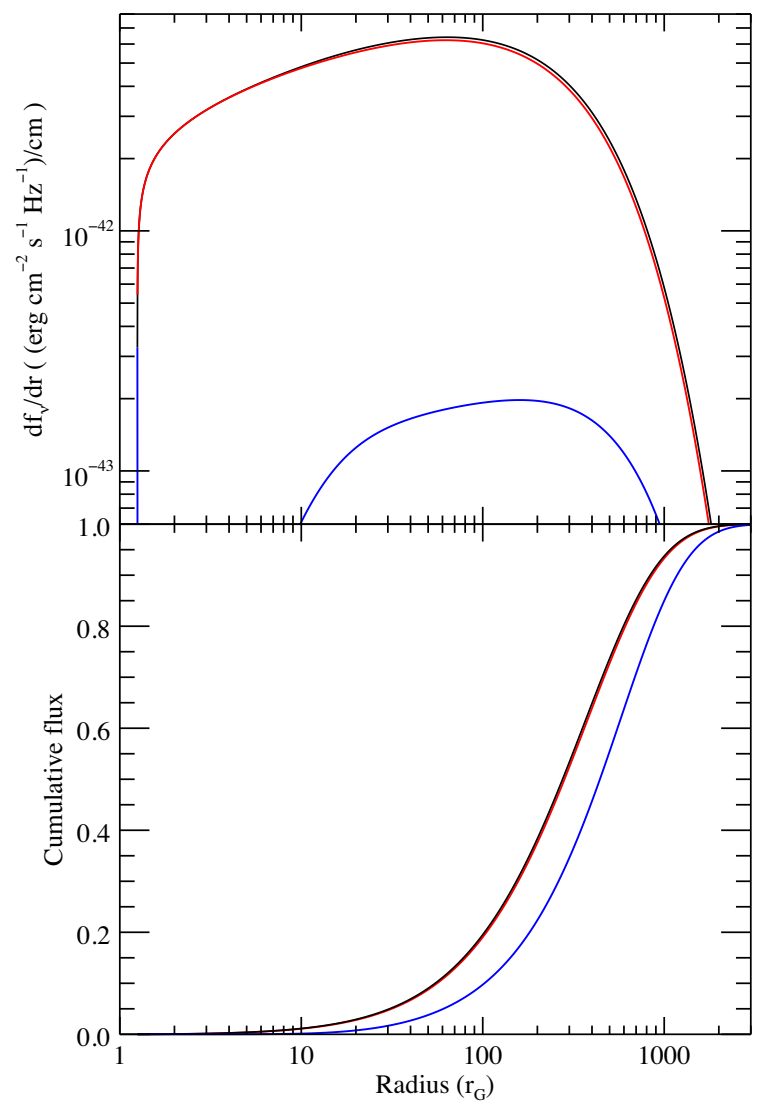

Figure 8. Emission at the central wavelength of the $W 1$ band from a standard disc with representative parameters for IRAS 13224-3809 (see the text for details). Black: without X-ray irradiation. Red: with X-ray irradiation. Blue: Difference.

sufficiently powerful to drive a significant fraction of the observed UV changes.

One alternative model to explain deviations from the simple $\mathrm{X}$-ray reprocessing scenario has been presented by Gardner \& Done (2017), in which a thickened hot inner disc acts as an intermediate reprocessor between the X-ray and UV emission. This has been suggested as an explanation for the correlations seen in NGC 5548 (Gardner \& Done 2017) and NGC 4151 (Edelson et al. 2017). While an additional reprocessor does not remove all correlation between $\mathrm{X}$-ray and UV flux, it significantly reduces the effect of fast X-ray variability on the UV emission. This could mean that X-ray/UV correlations are seen only on long time-scales and the campaign presented here is too short to detect a correlation.

The UV variability we do observe could still be due to illumination from the X-ray source if the variability seen by the disc is different to that in our line of sight. Various effects may lead to different X-ray variability being observed by the disc, such as variable absorption between the disc and corona. IRAS13224-3809 must have some outflowing material, which may shield the disc from the corona, as a highly ionized variable UFO is observed (Parker et al. 2017b). While this outflow is too optically thin to have a significant effect on the transmission of X-ray flux, optically thicker material (denser or less ionized) may exist in the acceleration zone, out of the line of sight, between the corona and disc. It is also possible for there to be a weak extended region of the corona which, although producing little X-ray flux, is optically thick when viewed from close to the plane of the disc. Scattering in this extended corona could significantly change the flux from the main central corona to the disc relative to that in our line of sight. If such material is present and changes within the observing campaign (which is seen by Parker et al. (2017b) to occur in the highly ionized material) then the X-ray flux which reaches the W1-band emitting region of the disc may not correlate with the observed X-ray flux.

Alternatively, the changes in X-ray intensity received by the disc may be different to those observed if the geometry of the system changes (such as the corona moving up and down) for several reasons. First, as the corona rises, it illuminates the disc from a less oblique angle, leading to stronger irradiation of the disc at constant coronal power. Additionally, if motion of coronal material is at relativistic speeds, changes of this motion will induce differences in the anisotropy of coronal emission due to special relativistic beaming. General relativistic light bending also acts to focus light towards the black hole (Miniutti \& Fabian 2004; Wilkins et al. 2016). While this principally affects the innermost regions, small effects in the outer regions may further complicate the observed variability. A combination of these effects along with changes in the intrinsic coronal power could lead to removal of the correlation between observed coronal power and UV emission from disc heating. The interpretation of the lack of correlation as being due to variable coronal geometry also fits with the relatively large X-ray variability of IRAS 13224-3809: if other sources have a more stable coronal geometry, they will be observed to have both weaker X-ray variability and stronger X-ray/UV correlation.

This interpretation could be tested with detailed mapping of the corona, such as in Wilkins \& Fabian (2011) and Wilkins \& Gallo (2015). This would allow the X-ray irradiation of the disc to be measured rather than just the X-ray flux in the line of sight. However, mapping the corona on sufficiently short time-scales is likely to require greater collecting area than is available with current missions.

\section{CONCLUSIONS}

We have shown that the X-ray and ultraviolet flux of the most X-ray variable bright AGN, IRAS 13224-3809, are not correlated on time-scales of up to $\sim 40 \mathrm{~d}$. However, the variability of the UV spectrum matches that seen in other AGN that do show X-ray/UV correlations. The UV variability is much weaker than in the X-rays: the average $W 1$-band fractional variability is $0.7 \pm 0.1$ per cent over one XMM-Newton orbit and around 3 per cent over $40 \mathrm{~d}$, whereas the X-rays vary by more than a factor of 10 on time-scales of kiloseconds. This suggests that the $\mathrm{X}$-ray variability viewed by the disc is different to that in our line of sight, which may be caused by changes in coronal geometry, absorption or scattering between the corona and outer disc.

\section{ACKNOWLEDGEMENTS}

We thank the referee for comments which have helped to improve the clarity of the paper. DJKB acknowledges financial support from the Science and Technology Facilities Council (STFC). ACF, AML, and MLP acknowledge support from the ERC Advanced Grant FEEDBACK 340442. BDM acknowledges support from the Polish National Science Center grant Polonez 2016/21/P/ST9/04025. This work has made use of observations obtained with XMM-Newton, an ESA science mission with instruments and contributions directly funded by ESA Member States and NASA. This work made use of data supplied by the UK Swift Science Data Centre at the University of Leicester. 


\section{REFERENCES}

Alston W. N., Vaughan S., Uttley P., 2013, MNRAS, 429, 75

Arévalo P., Uttley P., 2006, MNRAS, 367, 801

Arévalo P., Papadakis I., Kuhlbrodt B., Brinkmann W., 2005, A\&A, 430, 435

Boller T., Brandt W. N., Fabian A. C., Fink H. H., 1997, MNRAS, 289, 393

Buisson D. J. K., Lohfink A. M., Alston W. N., Fabian A. C., 2017, MNRAS, 464, 3194

Burrows D. N. et al., 2005, Space Sci. Rev., 120, 165

Cackett E. M., Horne K., Winkler H., 2007, MNRAS, 380, 669

Cackett E. M., Chiang C.-Y., McHardy I., Edelson R., Goad M. R., Horne K., Korista K. T., 2017, preprint (arXiv:1712.04025)

Chartas G., Kochanek C. S., Dai X., Moore D., Mosquera A. M., Blackburne J. A., 2012, ApJ, 757, 137

Chiang C.-Y., Walton D. J., Fabian A. C., Wilkins D. R., Gallo L. C., 2015, MNRAS, 446, 759

Dai X., Kochanek C. S., Chartas G., Kozłowski S., Morgan C. W., Garmire G., Agol E., 2010, ApJ, 709, 278

Davis S. W., Woo J.-H., Blaes O. M., 2007, ApJ, 668, 682

De Marco B., Ponti G., Uttley P., Cappi M., Dadina M., Fabian A. C., Miniutti G., 2011, MNRAS, 417, L98

De Marco B., Ponti G., Cappi M., Dadina M., Uttley P., Cackett E. M., Fabian A. C., Miniutti G., 2013, MNRAS, 431, 2441

Dewangan G. C., Boller T., Singh K. P., Leighly K. M., 2002, A\&A, 390, 65

Edelson R. A., Krolik J. H., 1988, ApJ, 333, 646

Edelson R., Turner T. J., Pounds K., Vaughan S., Markowitz A., Marshall H., Dobbie P., Warwick R., 2002, ApJ, 568, 610

Edelson R. et al., 2015, ApJ, 806, 129

Edelson R. et al., 2017, ApJ, 840, 41

Evans P. A. et al., 2007, A\&A, 469, 379

Evans P. A. et al., 2009, MNRAS, 397, 1177

Fabian A. C., Ross R. R., 2010, Space Sci. Rev., 157, 167

Fabian A. C. et al., 2009, Nature, 459, 540

Fabian A. C. et al., 2013, MNRAS, 429, 2917

Fausnaugh M. M. et al., 2016, ApJ, 821, 56

Gardner E., Done C., 2017, MNRAS, 470, 3591

Gehrels N. et al., 2004, ApJ, 611, 1005

Gibson R. R., Brandt W. N., Schneider D. P., 2008, ApJ, 685, 773

Gliozzi M., Papadakis I. E., Grupe D., Brinkmann W. P., Räth C., 2017, MNRAS, 464, 3955

Grupe D., Komossa S., Leighly K. M., Page K. L., 2010, ApJS, 187, 64

Haardt F., Maraschi L., 1991, ApJ, 380, L51

Haardt F., Maraschi L., 1993, ApJ, 413, 507

Jansen F. et al., 2001, A\&A, 365, L1

Kalberla P. M. W., Burton W. B., Hartmann D., Arnal E. M., Bajaja E., Morras R., Pöppel W. G. L., 2005, A\&A, 440, 775

Kara E., Cackett E. M., Fabian A. C., Reynolds C., Uttley P., 2014, MNRAS, 439, L26

Kara E., Alston W. N., Fabian A. C., Cackett E. M., Uttley P., Reynolds C. S., Zoghbi A., 2016, MNRAS, 462, 511

Leighly K. M., 2004, ApJ, 611, 125

Leighly K. M., Moore J. R., 2004, ApJ, 611, 107
Lightman A. P., White T. R., 1988, ApJ, 335, 57

Lobban A., Porquet D., Reeves J., Markowitz A., Nardini E., Grosso N., 2018, MNRAS, 474, 3237

Lohfink A. M., Reynolds C. S., Vasudevan R., Mushotzky R. F., Miller N. A., 2014, ApJ, 788, 10

Lyubarskii Y. E., 1997, MNRAS, 292, 679

Mason K. O. et al., 2001, A\&A, 365, L36

McHardy I. M. et al., 2016, Astron. Nachr., 337, 500

McHardy I. et al., 2017, preprint (arXiv:1712.04852)

Merloni A., Fabian A. C., 2003, MNRAS, 342, 951

Miniutti G., Fabian A. C., 2004, MNRAS, 349, 1435

Nandra K., George I. M., Mushotzky R. F., Turner T. J., Yaqoob T., 1997, ApJ, 476, 70

Pal M., Naik S., 2018, MNRAS, 474, 5351

Parker M. L. et al., 2017a, MNRAS, 469, 1553

Parker M. L. et al., 2017b, Nature, 543, 83

Ponti G. et al., 2010, MNRAS, 406, 2591

Ponti G., Papadakis I., Bianchi S., Guainazzi M., Matt G., Uttley P., Bonilla N. F., 2012, A\&A, 542, A83

Poole T. S. et al., 2008, MNRAS, 383, 627

Reis R. C., Miller J. M., 2013, ApJ, 769, L7

Robertson D. R. S., Gallo L. C., Zoghbi A., Fabian A. C., 2015, MNRAS, 453,3455

Roming P. W. A. et al., 2005, Space Sci. Rev., 120, 95

Shakura N. I., Sunyaev R. A., 1973, A\&A, 24, 337

Shappee B. J. et al., 2014, ApJ, 788, 48

Shemmer O., Brandt W. N., Netzer H., Maiolino R., Kaspi S., 2008, ApJ, 682,81

Smith R., Vaughan S., 2007, MNRAS, 375, 1479

Starkey D. et al., 2017, ApJ, 835, 65

Strüder L. et al., 2001, A\&A, 365, L18

Tanaka Y. et al., 1995, Nature, 375, 659

Timmer J., Koenig M., 1995, A\&A, 300, 707

Troyer J., Starkey D., Cackett E. M., Bentz M. C., Goad M. R., Horne K., Seals J. E., 2016, MNRAS, 456, 4040

Uttley P., Edelson R., McHardy I. M., Peterson B. M., Markowitz A., 2003, ApJ, 584, L53

Vagnetti F., Turriziani S., Trevese D., Antonucci M., 2010, A\&A, 519, A17

Vanden Berk D. E. et al., 2001, AJ, 122, 549

Vaughan S., Edelson R., Warwick R. S., Uttley P., 2003, MNRAS, 345, 1271

Wilkins D. R., Fabian A. C., 2011, MNRAS, 414, 1269

Wilkins D. R., Gallo L. C., 2015, MNRAS, 449, 129

Wilkins D. R., Cackett E. M., Fabian A. C., Reynolds C. S., 2016, MNRAS, 458, 200

Xu Y.-D., 2011, ApJ, 739, 64

Young A. J., Crawford C. S., Fabian A. C., Brandt W. N., O'Brien P. T., 1999, MNRAS, 304, L46

Zhou X.-L., Wang J.-M., 2005, ApJ, 618, L83

This paper has been typeset from a $\mathrm{T}_{\mathrm{E}} \mathrm{X} / \mathrm{LAT} \mathrm{E}$ file prepared by the author. 